\title{
Off-limb EUV observations of the solar corona and transients with the CORONAS-F/SPIRIT telescope-coronagraph
}

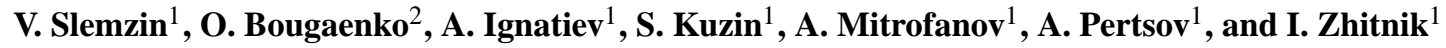 \\ ${ }^{1}$ P.N. Lebedev Physical Institute, Russian Academy of Sciences, Moscow, Russia \\ ${ }^{2}$ Sternberg Astronomical Institute, Moscow State University, Moscow, Russia
}

Received: 1 October 2007 - Revised: 25 January 2008 - Accepted: 25 January 2008 - Published: 15 October 2008

\begin{abstract}
The SPIRIT telescope aboard the CORONAS-F satellite (in orbit from 26 July 2001 to 5 December 2005), observed the off-limb solar corona in the $175 \AA$ (Fe IX, X and XI lines) and $304 \AA$ (He II and Si XI lines) bands. In the coronagraphic mode the mirror was tilted to image the corona at the distance of $1.1 \ldots 5 R_{\text {sun }}$ from the solar center, the outer occulter blocked the disk radiation and the detector sensitivity was enhanced. This intermediate region between the fields of view of ordinary extreme-ultraviolet (EUV) telescopes and most of the white-light (WL) coronagraphs is responsible for forming the streamer belt, acceleration of ejected matter and emergence of slow and fast solar wind. We present here the results of continuous coronagraphic EUV observations of the solar corona carried out during two weeks in June and December 2002. The images showed a "diffuse" (unresolved) component of the corona seen in both bands, and non-radial, ray-like structures seen only in the $175 \AA$ band, which can be associated with a streamer base. The correlations between latitudinal distributions of the EUV brightness in the corona and at the limb were found to be high in $304 \AA$ at all distances and in $175 \AA$ only below $1.5 R_{\text {sun }}$. The temporal correlation of the coronal brightness along the west radial line, with the brightness at the underlying limb region was significant in both bands, independent of the distance. On 2 February 2003 SPIRIT observed an expansion of a transient associated with a prominence eruption seen only in the $304 \AA$ band. The SPIRIT data have been compared with the corresponding data of the SOHO LASCO, EIT and UVCS instruments.
\end{abstract}

Keywords. Solar physics, astrophysics, and astronomy (Corona and transition region; Ultraviolet emissions; Instruments and techniques)

Correspondence to: V. Slemzin

(slem@sci.lebedev.ru)
1 Observations of the solar corona by wide field instruments

The solar corona is routinely observed now with two types of instruments: the temperature sensitive, space-based Xray and EUV telescopes (e.g. SOHO EIT, Delaboudinière et al., 1995), to study the inner corona up to $R \sim 1.3$ (hereafter $R$ is the distance from the solar center in the units of the solar radius) and the electron density sensitive space and ground-based WL coronagraphs to study the outer corona above $R=2$. Excellent examples of ground-based observations of the corona, starting from the limb level during the solar eclipses can be found elsewhere (Koutchmy, 1994; Wang et al., 2007) but the solar eclipses are infrequent and last only a few minutes. Most of the data have been obtained up to now with the SOHO LASCO C2 and C3 coronagraphs (Brueckner et al., 1995). The LASCO C1 coronagraph observed the corona in the green line in the intermediate range $R=1.1 \ldots 3.2$ (Schwenn et al., 1997) only at the solar minimum in 1996-1998.

Recently, the upper limit of the regular coronal observations with the EUV telescopes was increased to $R=1.7$ (STEREO SECCHI EUVI, Wülser et al., 2004) and the lower limit for the space-based WL coronagraphs reduced to $R=1.3$ (STEREO SECCHI COR-1, Thompson and Davila, 2007). The ground-based MK4 coronagraph at Mauna Loa Solar Observatory is able now to observe the corona from $R=1.1$ (Burkepile et al., 2005).

In numerous observations it was determined that the inner corona consists of a "diffuse" part, possibly formed by many unresolved loops and well described by a hydrostatic approximation, and bright, raylike or threadlike axisymmetric and non-axisymmetric structures of different spatial scales, forming a base of streamers (e.g. Koutchmy, 2001; Koutchmy and Molodensky, 2005). The structure and local parameters of the coronal plasma in the EUV range at distances below $R=1.6$ were studied by many researchers (e.g. Wilhelm et

Published by Copernicus Publications on behalf of the European Geosciences Union. 
al., 1998, 2002; Warren, 1999; Parenti et al., 2000; Li et al., 2000; Cirtain et al., 2006; DeForest, 2007, and others). The parameters of the outer corona above $R=2$, mainly the electron density and its 3-dimensional distribution, were retrieved from the observations with the WL coronagraphs (e.g. Lamy et al., 1997; Quemerais and Lamy, 2002; Saez et al., 2005). The intermediate region between $R=1.6$ and 2 has not been studied in detail, in particular, in the EUV, although it is essential for theoretical modelling of streamers and solar wind generation. Besides the problem of physical matching of the EUV and WL data, it seems to be important to extend the X-ray and the EUV measurements from the disk to at least $R=2 \ldots 3$, with similar spatial and temporal resolution to watch spatial and temporal variations of the plasma structure and the development of Coronal Mass Ejections (CMEs).

The ability of an ordinary EUV telescope to register a coronal brightness at the distances larger than $R=1.3$ is limited by the dynamic range of the detector based on Charge Coupled Devices (CCD), which is typically about 4.5 orders of magnitude, and the straylight level ( $\sim 1 \%$ of the mean intensity at the disk). Delaboudinière (1999) was the first to overcome the latter limitation and observed the corona with the EIT telescope in the equatorial plane to the distances $R \sim 2.6$. These observations were made while the spacecraft was in the offset position and the solar disk radiation was blocked by a mask in front of the detector.

The solar corona at $R=1.3 \ldots 12$ is studied in the Far UV spectral range with the Ultraviolet Coronagraph Spectrometer aboard the SOHO observatory (Kohl et al., 1995; Raymond et al., 1997). This instrument has two UV spectral channels: the OVI channel operating in the range 945$1123 \AA(473-561 \AA$ in the second order), and the Ly $\alpha$ channel operating in the range 1160-1350 $\AA$ (580-635 $\AA$ ). The images are obtained in the discrete spectral lines Ly $\alpha$ $1216 \AA$, FeXII $1242 \AA$, O VI $1032 \AA$, Si XII 499/521 Å, Mg $\mathrm{X} \mathrm{610/625 \AA} \mathrm{and} \mathrm{some} \mathrm{others} \mathrm{in} \mathrm{the} \mathrm{slit-like} \mathrm{fields} \mathrm{of} \mathrm{view}$ (FOV) of $14^{\prime \prime} \times 40^{\prime}$ (Ly $\alpha$ channel) and of $28^{\prime \prime} \times 40^{\prime}$ (O VI channel). In the standard synoptic mode a panoramic view of the corona is constructed by a series of discrete exposures at 8 angular and up to 12 radial positions with the total observational time of $\sim 20 \mathrm{~h}$. UVCS is a very effective diagnostic device for the analysis of local ionization equilibrium or line-of-sight velocity distributions, but cannot study spatial and temporal dynamics of the coronal structures in a wide field of view.

The SPIRIT EUV telescope-coronagraph is a new instrument for wide-field observations of the solar disk and the corona in the 175 and $304 \AA$ EUV spectral bands (Zhitnik et al., 2002). The instrument is able to observe wide segments (more than $90^{\circ}$ in latitude) of the solar corona from the limb to $R=5$ with high spatial $\left(5^{\prime \prime}\right)$ and temporal $(5 \mathrm{~min}$ ) resolution, which has no analogues in the practice of the solar astronomy. The main task for the CORONAS flight was to prove the concept and the design of such instrument and to define its potential for studying the solar corona and tran- sients. In the period of high solar activity (2002-2003) the SPIRIT EUV telescope operated in the coronagraphic mode during 15 observation sessions lasting from several hours to one week. The current report presents the main parameters of the instrument and some significant results of the SPIRIT observations in comparison with the data of SOHO LASCO, EIT and UVCS.

\section{SPIRIT EUV telescope-coronagraph}

\subsection{Design of the instrument and the observation procedure}

One of the two SPIRIT EUV telescopes had two channels operating in 175 and $304 \AA$. It had been designed according to the Herschel optical configuration which provides a good angular resolution and the largest possible efficiency due to only one reflection. The distance between the offaxis parabolic mirrors and the detectors in SPIRIT was three times larger than in the Ritchey Chretien telescopes with similar resolution (e.g. SOHO/EIT, SECCHI/EUVI), so the straylight in our case was at least one order less. In order to operate as a coronagraph, the telescope was provided with drivers to tilt the mirror up to $+/-1.5^{\circ}$ from its nominal position (Zhitnik et al., 2003). The optical design of the SPIRIT telescope-coronagraph is shown in Fig. 1a. A driven lid in front of the input window was used as an outer occulter to block the direct illumination of the mirror by the intense solar disk radiation. The corona was observed through additional lateral optical windows. Both the central and the lateral windows have been covered with similar thin film filters. The detector had an image intensifier with a variable amplification, a lens and a CCD-array for the registration of visible images. The exposure time in the disk mode was $9 \mathrm{~s}$, in the coronagraphic mode $300 \mathrm{~s}$. Due to longer exposures and higher amplification of the detector, a sensitivity of the instrument in the coronagraphic mode was 143 times (in the $175 \AA$ band) and 316 times (in the $304 \AA$ band) more than in the disk mode. It should be noted that in both modes the exposures in the 175 and $304 \AA$ bands were strictly simultaneous.

Due to the telescope design restrictions, the occulters were mounted eccentrically to the telescope optical axis, so the viewing range in the coronagraphic mode depended on the current position of the FOV towards the instrumental axes. The FOV had the geometrical size of $46^{\prime} \times 52^{\prime}$. In total, the instrument was able to observe the corona from the limb to the radial distance of $R=5$. The orientation of the FOV with regard to the solar axes varied in time, due to a slow roll of the satellite (ordinarily the roll velocity was less than 3$4^{\circ} 1 /$ day). The roll angle was not stabilised, but its value was recovered on ground using the attendant pictures of the sky taken by two star cameras in the SPIRIT assembly. The vignetting function of the coronagraph (Fig. 1b) had a steep rise from the limb to $R=4$, so it acted as a radial filter 

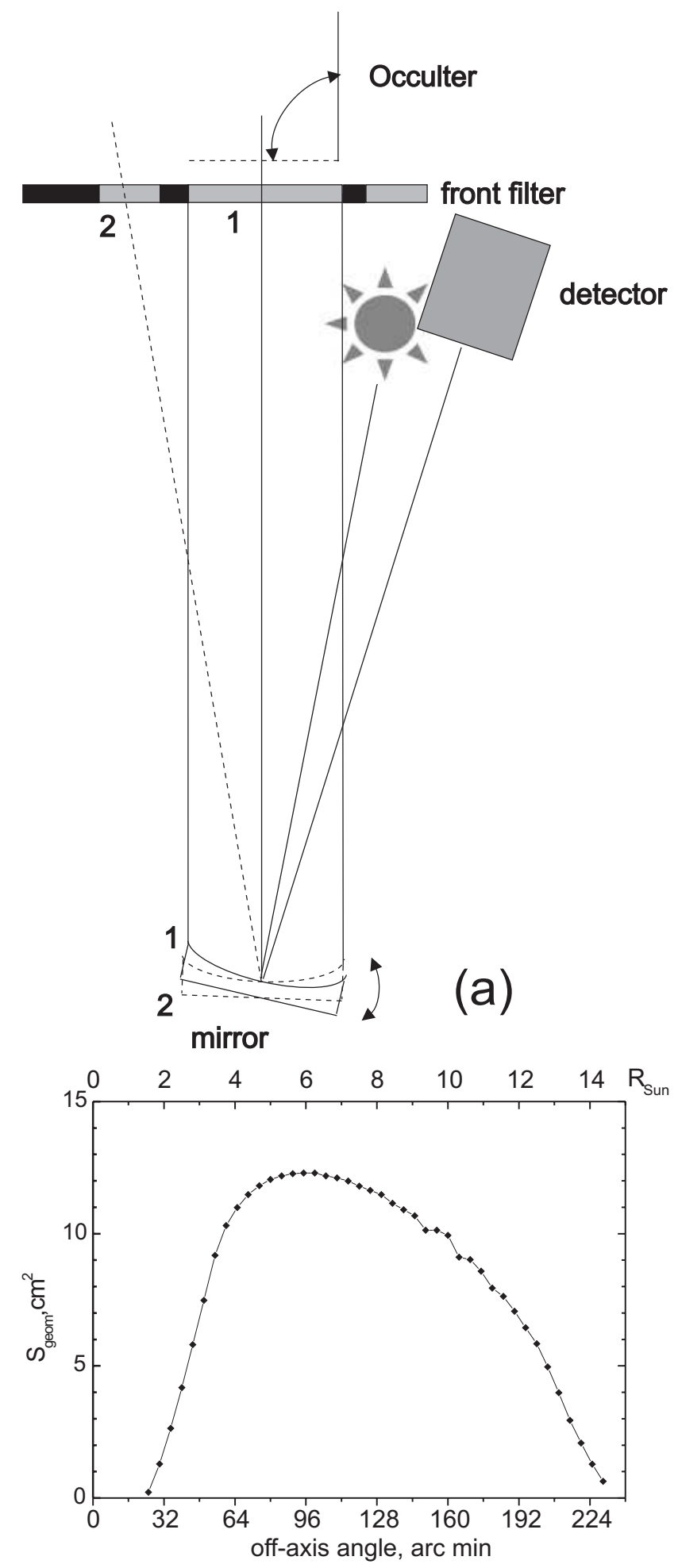

Fig. 1. (a) Optical design of the SPIRIT telescope-coronagraph and the ray paths in the disk mode (1) and in the coronagraphic mode (2); (b) the geometrical area of the coronagraph versus the off-axis angle (a vignetting function) with the closed occulter.
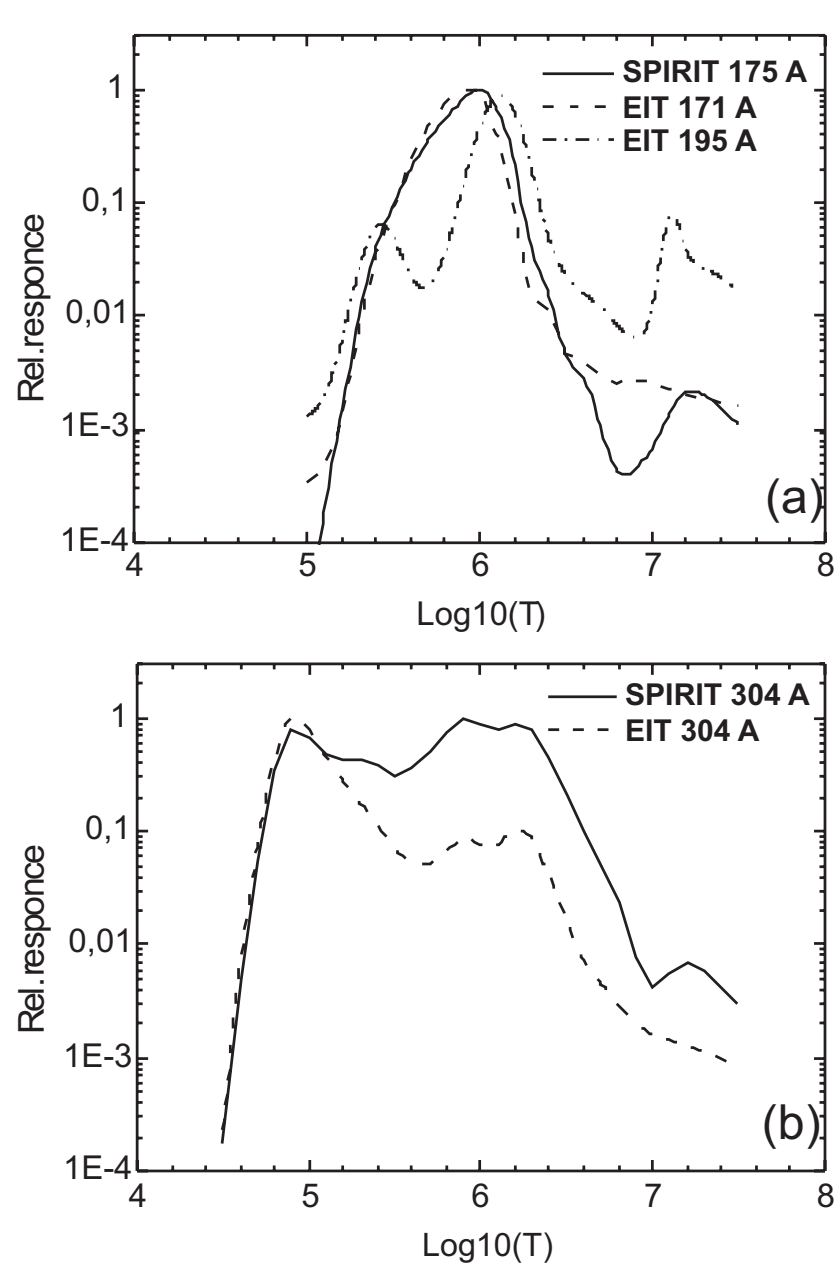

Fig. 2. Plasma temperature response functions for the SPIRIT $175 \AA$ channel (a) and the $304 \AA$ channel (b) compared with the functions for the corresponding EIT channels 171/195 and $304 \AA$.

analogous to that used in the WL coronagraphs, to equalize the coronal signal at different heights.

The observational cycle consisted of three steps. First, the mirror was tilted in order to place the FOV into the preprogrammed position relative to the solar disk (taking into account the current roll angle). Then the shifted image of the disk was registered in the disk mode with the open occulter, to control the real position of the FOV towards the Sun. Then the occulter was closed, and several images of the corona were taken in the enhanced coronagraphic mode. As a rule, images of the disk were taken every $2-3 \mathrm{~h}$, between those from 4 to 12 coronal images were taken with a cadence of 15-30 min. Ordinarily, the images in the coronagraphic mode were transmitted to the Earth in the binned mode with the pixel size of 5.4", with the total number of frames and the image format being limited by the available telemetry volume. Blurring of the coronal images produced by the drift 

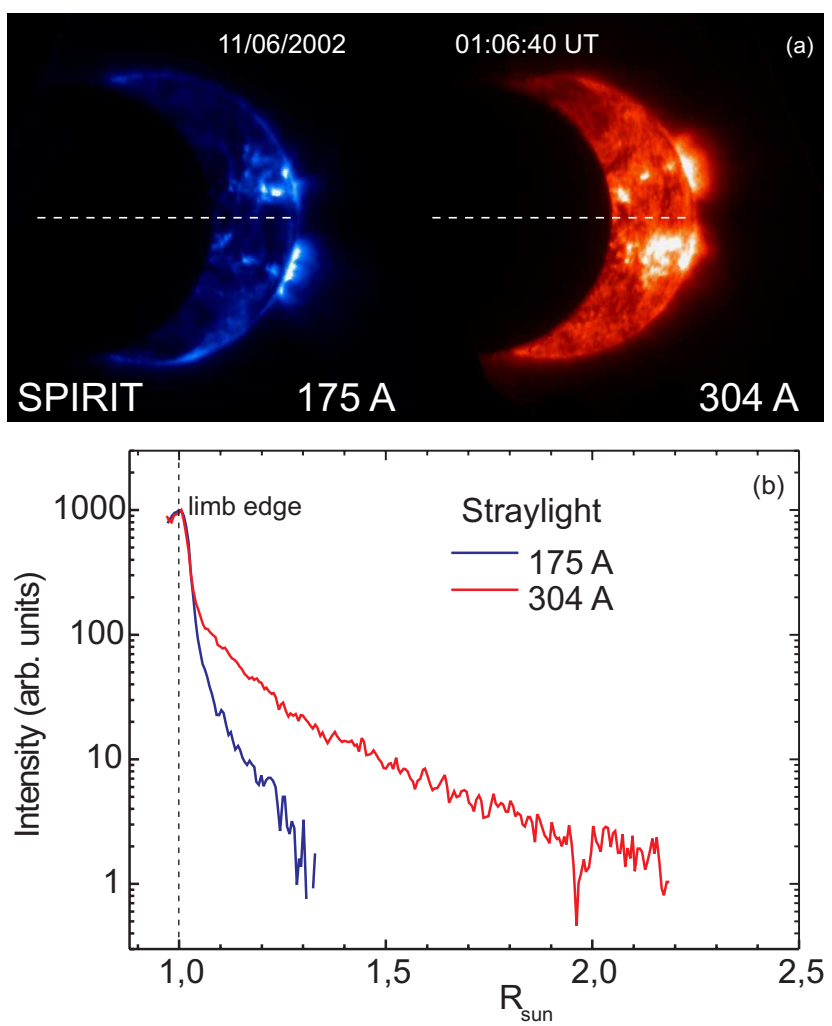

Fig. 3. (a) SPIRIT images in 175 and $304 \AA$ obtained during the eclipse of 11 June 2002, 01:06:40 UT; (b) radial distributions of the straylight in the SPIRIT 175 and $304 \AA$ bands along the scan directions marked by the dashed lines.

of the satellite pointing system and variation of the roll angle during the exposure time did not exceed $10-15^{\prime \prime}$.

2.2 Plasma temperature response functions for the 175 and $304 \AA ̊$ bands

Figure $2 \mathrm{a}$ and $\mathrm{b}$ presents the plasma temperature response functions (PTR) of the SPIRIT 175 and $304 \AA$ bands in comparison with the similar channels of the SOHO/EIT telescope (Slemzin et al., 2005). The SPIRIT $175 \AA$ channel had the PTR similar to that of the $171 \AA$ EIT channel. Calculations with the use of the CHIANTI code (Dere et al., 1997) showed that more than $80 \%$ of its input was provided by three lines of the Fe IX, X and XI ions ( $\left.T_{\max } \sim 0.6 \ldots 1.5 \mathrm{MK}\right)$. The PTR of the SPIRIT $304 \AA$ channel had two peaks: the major peak corresponded to the He II line $\left(T_{\max } \sim 80.10^{3} \mathrm{~K}\right)$ and the minor peak at $T \sim 1 \ldots 2 \mathrm{MK}$, associated with the coronal Si XI 303.3 $\AA$, Fe XV 284.2 and Fe IX 171.1 $\AA$ lines (the latter registered in the second reflection order of the mirror multilayer coating). Estimations with the help of the CHIANTI code showed that in quiet solar regions, more than $50 \%$ of the total signal in the SPIRIT $304 \AA$ band corresponded to the $\mathrm{He}$ II $304 \AA$ line. The response functions of the SPIRIT and EIT
$304 \AA$ bands are similar except for the larger contribution of the hot ion lines, in our case, caused by a wider spectral function. The contribution of the hot component can be easily distinguished by a comparison of the 304 and $175 \AA$ images, just as Delaboudinière (1999) did for the EIT images.

\subsection{Processing and calibration of the images}

The images of the disk and corona were first pre-processed using a standard procedure which included a background subtraction, a correction of nonlinearity of the brightness scale (appeared at the highest amplification), and a flat-field correction. Using the data of the star cameras the images were rotated to compensate for the roll of the satellite, in order to fit the Y-axis of the image with the solar rotation axis. In some cases the images of the corona were summed over the period of several hours for a better signal-to-noise ratio. Then the images of the corona were superimposed on the nearest disk image to build a composite image. To perform a photometric analysis, the coronal images were calibrated by dividing the signal in each pixel to the value of the vignetting function according to its radial position and then the disk and coronal parts were matched at the distance of $R=1.3$.

The angular distribution of the straylight was studied during several partial solar eclipses observed with SPIRIT from the CORONAS-F orbit. Figure 3 a shows the images taken during the solar eclipse of 11 June 2002; Fig. $3 \mathrm{~b}$ - the straylight radial functions for the 175 and $304 \AA$ A bands. The straylight functions were sufficiently narrower and steeper than the measured radial brightness distributions of the corona. In the analysis presented here a contribution of the straylight was neglected.

\section{Results}

3.1 Movies of the solar corona in the $175 \AA$ and $304 \AA$ bands

The movies Mv01 and Mv02 (online supplementary material at http://www.ann-geophys.net/26/3007/2008/ angeo-26-3007-2008-supplement.zip) were composed using the SPIRIT images taken in the 175 and $304 \AA$ bands during two sessions of continuous observations in June and December 2002. Mv01 shows the west region within -60 to $+40^{\circ}$ (relative to the east-west line) observed on 16 June 2002...22 June 2002. Mv02 shows the east region observed on 3 December 2002...10 December 2002. In order to enhance the coronal structures, the disk and the coronal part of the images were presented in a logarithmic scale and separately scaled in intensity relative to the maximum brightness. Figure 4 shows the three selected frames from the movies demonstrating the common features observed in the corona.

In the $175 \AA$ band (Fe IX-XI lines) the corona contained a "diffuse" (unresolved), quasi-symmetric part and a structure 
of bright rays starting from the solar surface, some of them deviated from the initial direction of tens degrees at the distance $R=1.3-1.5$ (examples in Fig. 4: the radial rays are marked by the number 1 , the non-radial ones by the number 2). Some very bright rays which evidently originated from the active regions appeared near the limb (e.g. a group of rays in the northeast sector marked by the number 4 in Fig. 4, seen on 21 June 2002...22 June 2002), but it is not obvious for all cases. Possibly, these rays correspond to open magnetic structures associated with the solar wind streams. Several big rising loops (e.g. feature 3 in Fig. 4) were seen and have been identified with the CMEs in the LASCO catalogue http://cdaw.gsfc.nasa.gov (e.g. CMEs of 16 June 2002 15:50:07; 17 June 2002 21:20:06; 20 June 2002 22:30:32; 6 December 2002 13:31:47).

In $304 \AA$ the corona was inhomogeneous and more "diffuse" than in $175 \AA$. Only traces of the coronal rays seen in $175 \AA$ were faintly visible in this band. The brightness in the corona was higher when some bright regions appeared at the disk near the limb (or behind it).

\subsection{Comparison of SPIRIT images with LASCO and UVCS}

Figure 5a-d shows the composite pictures of the SPIRIT images and the LASCO C2 images for the same temporal periods of 16 June 2002 and 22 June 2002. The SPIRIT images of the corona were summed over 9-h intervals of observations. The contrast of the SPIRIT coronal images has been enhanced, as explained in the previous section.

The rays seen in the SPIRIT $175 \AA$ picture (Fig. 5a and c) between the limb and the inner boundary of the LASCO FOV ( $R=2.3$ ) marked by the numbers from 1 to 6 evidently have counterparts in the streamer structure seen in the LASCO images. It is worth keeping in mind that the coronal brightness in the LASCO images is proportional to the total electron density $n_{e}$ of the plasma, whereas in the $175 \AA$ band it is proportional to $n_{e}^{2}$ of the plasma component with the temperature near $1 \mathrm{MK}$.

The correlation between the latitudinal distributions of the coronal brightness in the SPIRIT $304 \AA$ and LASCO images (Fig. 5b and d) is not high or even negative: some of the brightest features in $304 \AA$ at $R=1.1 \ldots 1.5$ correspond to the regions of weaker brightness in the LASCO picture at $R>2.3$ and vice versa.

Figure 6 demonstrates a comparison of the SPIRIT and UVCS images for 16 June 2002: (a) the SPIRIT image in $175 \AA$ from the limb to $R=1.6$ superimposed on the UVCS image ( $R=1.6 \ldots 3.5)$ in the OVI line (1032 $\AA$ ); (b) the SPIRIT image in $304 \AA$ superimposed on the UVCS image in the Ly $\alpha$ line $(1216 \AA)$. In the first case only some of the rays seen in $175 \AA$ (e.g. features numbered as 1 and 2 in Fig. 6a) have counterparts in the UVCS images. The difference in contrast to the coronal structures seen in the SPIRIT and UVCS images can be explained by their supposed mechanisms of

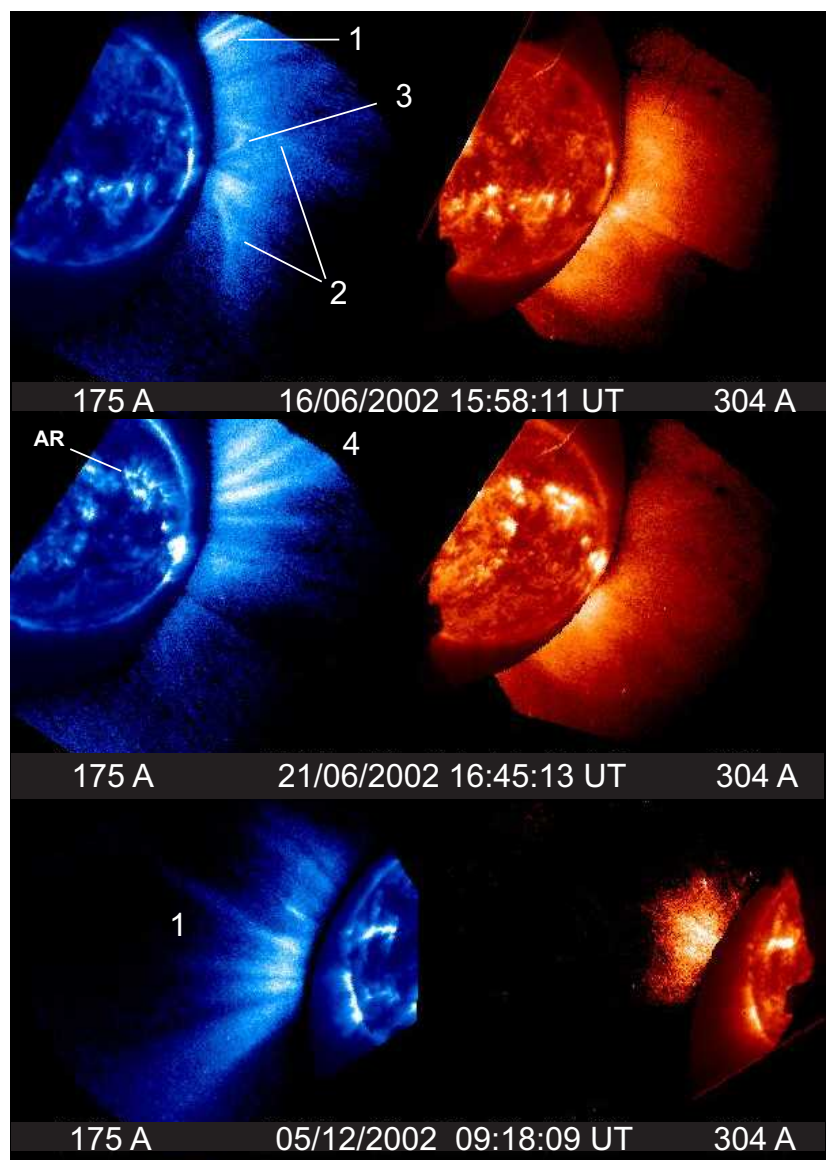

Fig. 4. Specific coronal features observed by the SPIRIT EUV coronagraph in the 175 and $304 \AA$ bands: 1 - radial rays, 2 - non-radial rays, 3 - a rising loop, $4-\mathrm{a}$ fan of rays linked with the active region at the disk.

excitation. Whereas the $\mathrm{Fe}$ ion lines of the $175 \AA$ band are excited by collisions, in the O VI line the collisional and radative components are of one order of value (Raymond et al., 1997). Besides, the collisional components in the $\mathrm{Fe}$ IX-X-XI and O VI images correspond to different excitation temperatures: $T_{e} \sim 1 \mathrm{MK}$ and $0.3 \mathrm{MK}$, correspondingly.

The corona in the pair of the SPIRIT $304 \AA$ and the UVCS Ly $\alpha 1216 \AA$ images (Fig. $6 \mathrm{~b}$ ) do not show the structural elements seen in the SPIRIT $175 \AA$ Amage. The angular distributions of brightness in both cases are roughly similar: the brightness in the sectors numbered by 1 is higher than those in sector 2 in both bands. It corresponds to the suggestion that the coronal radiation in both cases is generated by the same mechanism of resonant scattering. The Ly $\alpha$ line is less sensitive to the line-of-sight velocity than the He II line (Kohl et al., 1995). 


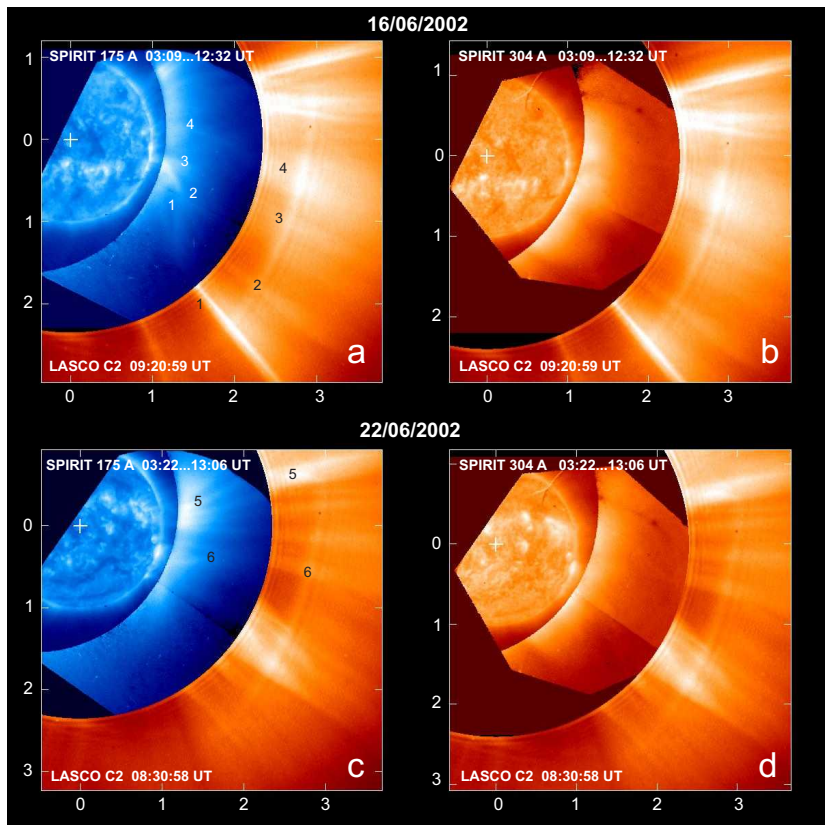

Fig. 5. Composite SPIRIT - LASCO C2 images: (a) and (c) the SPIRIT 175 image at $R=0 . .2 .3$ with the LASCO C2 image (at $R>2.3$ ), (b) and (d) the SPIRIT $304 \AA$ image with the LASCO C2 image. Panels (a) and (b) show the SPIRIT images for 16 June 2002 summed over the period of 03:09...12:32 UT, and the LASCO image taken on the same day at 09:20:59 UT. Panels (c) and (d) show the SPIRIT images for 22 June 2002 summed over the period of 03:32...13:06 UT, and the LASCO image taken on the same day at 08:30:58 UT. The scales are in units of the solar radius (the origin marked by a cross corresponds to the solar center). The pair numbers $1 . . .6$ designate the features which probably belong to the same structural elements seen in EUV and WL.

3.3 Spatial structure and temporal variability of EUV brightness in the corona at the distances of $R=1 \ldots 2$

Figure 7a shows the latitudinal distributions of the coronal brightness at different radial distances in both SPIRIT bands for 16 June 2002 (the west direction). In order to enhance the signal-to-noise ratio, the initial SPIRIT images of the corona were summed over the period of 03:09...12:32 UT, and the resulting latitudinal distributions were averaged over $1^{\circ}$ bins. The limb distributions were taken from the disk images at 09:19:51 UT. Figure 8a demonstrates the maximal values of the cross-correlation functions between the latitudinal brightness distributions and the reference limb distribution in each band obtained with all possible latitudinal lags. Whereas at all distances the distributions in $304 \AA$ correlate well with the corresponding limb distribution, in the $175 \AA$ the correlation at $R>1.5$ drops below 0.5 , reflecting the non-radial structure of the corona at these distances.

The results may be interpreted in terms of the excitation mechanisms of the Fe and He ion lines. Due to the collisional excitation, at distances of $R=1 \ldots 1.5$, the coronal brightness
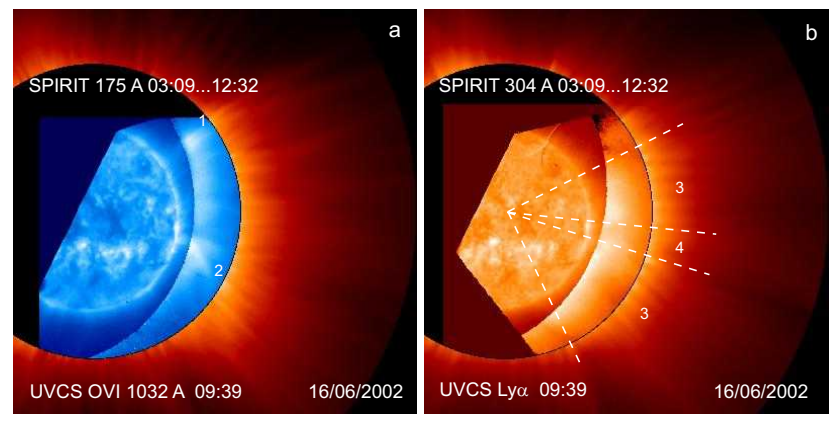

Fig. 6. Composite SPIRIT (at $R=1 . .1 .6$ ) and the UVCS images (at $R=1.6 \ldots 3.5$ ): (a) SPIRIT $175 \AA$ and UVCS OVI $1032 \AA .1$ and $2-$ the rays in the SPIRIT having the counterparts in the UVCS image; (b) SPIRIT $304 \AA$ and UVCS Ly $\alpha$. In both images the brightness in sector 3 is relatively higher than in sector 4 .

in the $175 \AA$ band is the largest in the relatively dense closed loops localized above the active regions. These structures cannot be resolved with our spatial resolution $\left(1^{\circ}\right.$ bin corresponds to $1.2 \times 10^{4} \mathrm{~km}$ at the limb), but their averaged brightness is proportional to the brightness of the active regions at the limb. At larger distances the major brightness is concentrated in non-radial rays, so the correlation vanishes.

In the $304 \AA$ band the main mechanisms of excitation of Helium are: collisional excitation in the closed loops near the limb (probably enhanced by the non-thermal electrons or ionic diffusion) with the minor contribution of the radiative excitation by the photoionization-recombination (P-R) process (Zirin, 1975; Macpherson et al, 1999; Andretta et al., 2003; Judge et al., 2004). Above the limb this band also contains a contribution of the collisionally excited Si XI and Fe XV lines. In total, these components result in a positive correlation between the coronal brightness in the $304 \AA$ band with the brightness at the limb. At the distances $R>1.5$ the electron density is low, and the radiative excitation, namely, the resonance scattering becomes dominant (Delaboudinière, 1999), which also provides a positive correlation with the brightness at the limb.

Figures $7 \mathrm{~b}$ and $8 \mathrm{~b}$ show temporal variations of the coronal brightness along the west equatorial line during the week 16 June 2002-22 June 2002, normalized to unity at each distance and their correlations with the limb distribution. The scan line does not cross any bright coronal rays and corresponds to the "diffuse" corona above the quiet solar region.

In contrary with latitudinal distributions, the temporal variations are well correlated with those at the limb in both bands, independent of the distance. This result confirms that the corona at the distances $R=1 \ldots 2$ is closely linked with the brightness distribution in the underlying disk regions, varying with the solar rotation. 

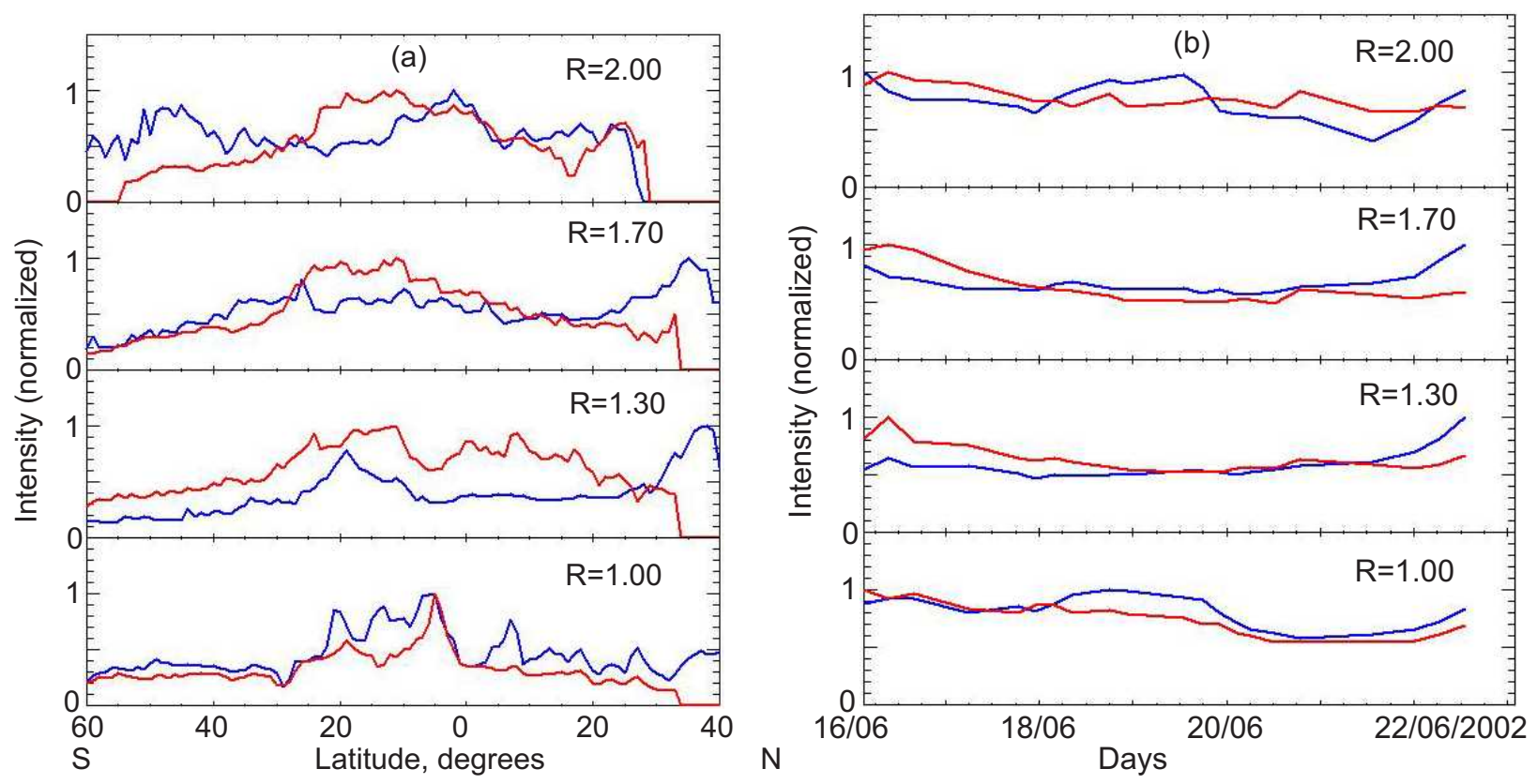

Fig. 7. (a) Latitudinal distributions of the corona brightness above the west limb for 16 June 2002; (b) temporal variations of the corona brightness at different distances along the west equatorial line for the period 16 June 2002...22 June 2002. The blue lines correspond to $175 \AA$, the red lines - to $304 \AA$. The intensities are normalized to the maximum values for each distance.

\subsection{Radial distributions at the equatorial plane}

We analyzed the radial distributions of the EUV coronal brightness in the equatorial plane using the SPIRIT data of 16 June 2002 described in the previous section. Figure 9 shows the normalized radial distributions in the SPIRIT 175 and $304 \AA$ bands (relative to the brightness at the limb) compared with the EIT data taken from the paper of Delaboudinière (1999). These data were obtained on 4 April 1996 when the SOHO spacecraft was in the off-set position, and presented as normalized radial brightness distributions in the $\mathrm{Fe}$ ion lines (an averaged distribution of practically coincident data in the 171, 195 and $284 \AA$ bands) and in the $304 \AA$ band along the east-west line in the eastern direction. It should be noted that the SPIRIT data correspond to the maximum of the solar activity, whereas the EIT data were taken at the minimum of the activity.

There are three important conclusions which result from Fig. 9.

1. The normalized radial distribution in $304 \AA$ from the SPIRIT data coincides with the corresponding one in $175 \AA$ in the region $R=1 \ldots 1.8$. The EIT distributions in $304 \AA$ and in the coronal $\mathrm{Fe}$ lines also coincide in the region $R=1 \ldots 1.2$. It suggests that in these regions the emission in the $304 \AA$ band (which consists of the major He II line and accompanying Si XI and Fe XV lines) has the same dependence on the coronal electron density as the corresponding emissions in the Fe bands which are excited by electron-ion collisions. The region of predominantly collisional excitation of the $\mathrm{He}$ II line is larger at the solar maximum (the SPIRIT case) in comparison with that at the solar minimum (the EIT case) because of higher electron density in the middle corona at the solar maximum.

2. At the distances larger than $R=1.8$ (in the SPIRIT case) and $R=1.2$ (in the EIT case) the radial distributions in the SPIRIT and EIT $304 \AA$ A bands are shallower than the corresponding Fe line distributions. At the distances of $R>2$ the SPIRIT and EIT $304 \AA$ distributions practically coincide. According to the assumption of Delaboudinière (1999), it means that in these regions the resonant scattering of the disk radiation dominates in the He II line emission of the corona.

3. A comparison between the SPIRIT and EIT radial distributions shows the variation of the coronal EUV emission in the equatorial plane with the solar activity: in the range $R=1.1 \ldots 2.5$ the normalized distribution at the solar maximum sufficiently exceeds that at the solar minimum and approaches it at larger distances.

A detailed quantitative analysis of radial distributions of the coronal EUV emission from the SPIRIT data will be done in further works. 

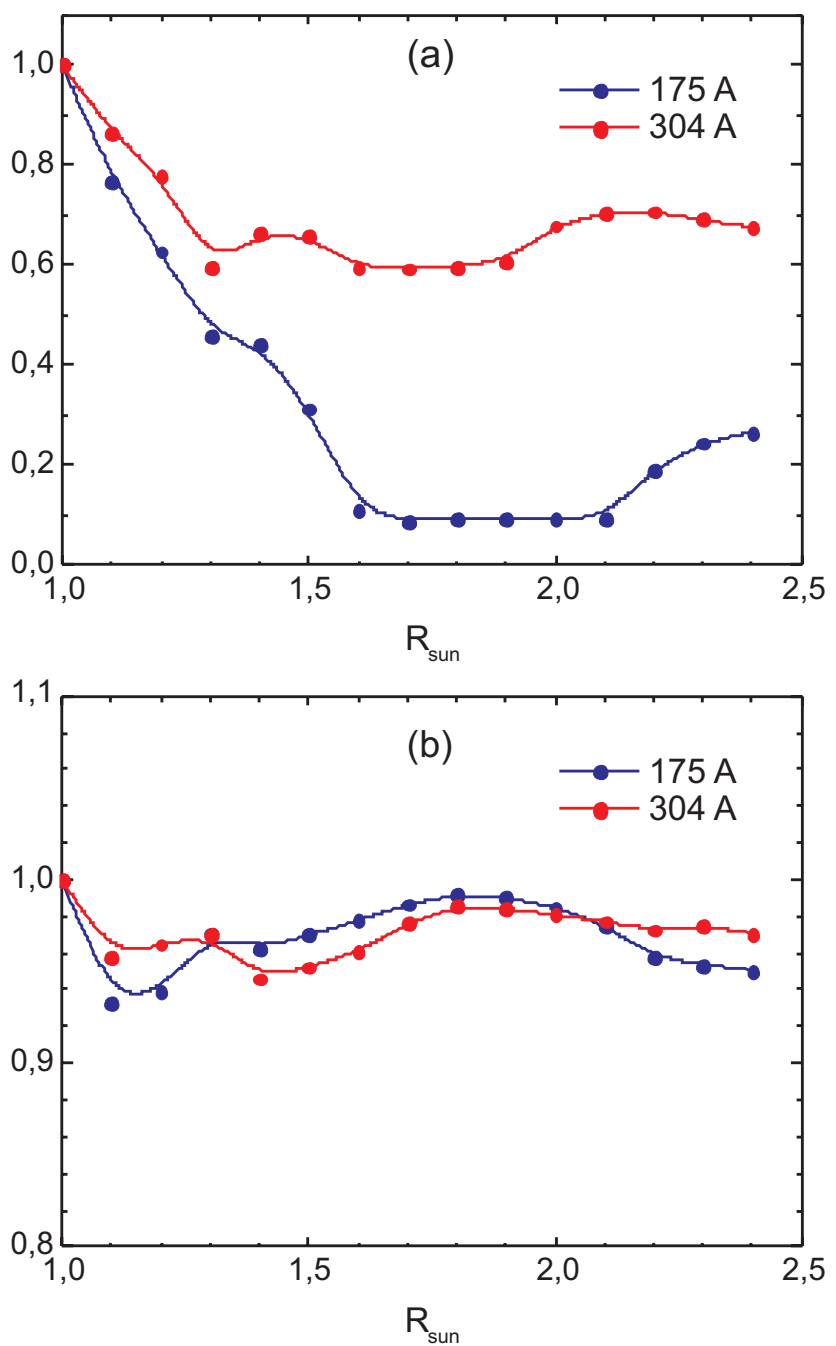

Fig. 8. (a) Cross-correlation between the latitudinal brightness distributions for different distances in the west corona and the limb distributions in the SPIRIT 175 and $304 \AA$ bands for 16 June 2002; (b) cross-correlation of the temporal brightness variations at different heights along the west equatorial line with that at the underlying limb region during a week of 16 June 2002-22 June 2002.

\subsection{Observations of transients in the middle corona}

During the SPIRIT coronagraphic observations we detected several transients associated with eruption processes and coronal mass ejections (CMEs). Simultaneous observations in two spectral channels associated with different plasma temperatures give unique information to test and improve the theoretical models of the eruption processes. As an example, Fig. 10 demonstrates the selected pictures of the CME after a failed prominence eruption observed on 2 February 2003 between 13:00 and 18:00 UT. To enhance the contrast of transients, the coronal parts are shown as the base difference images obtained by the subtraction from each image of the

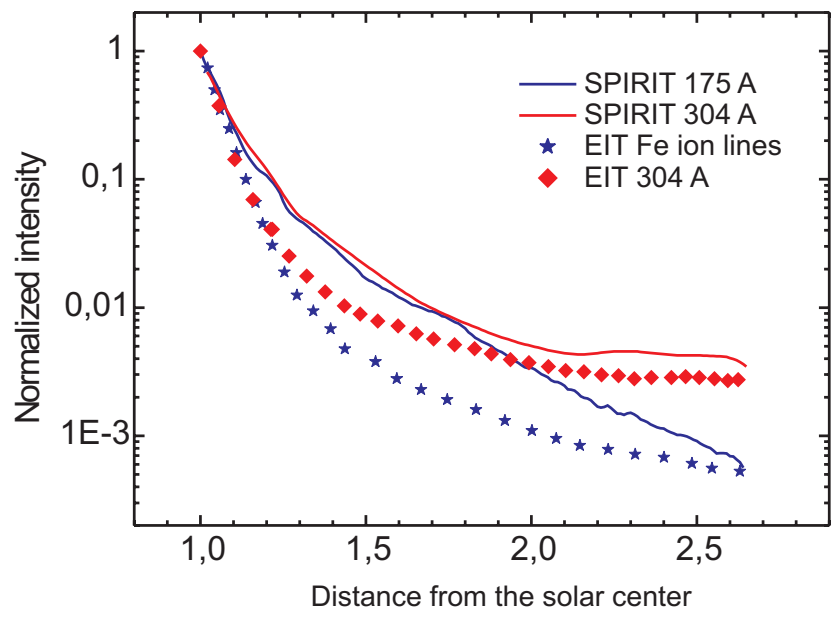

Fig. 9. Normalized radial distributions of the EUV coronal brightness with the distance at the equatorial plane: solid blue and red lines - the SPIRIT data for the west equatorial line taken on 16 June 2002 in the 175 and $304 \AA$ bands correspondingly, red diamonds and blue stars - the EIT data for the east equatorial line taken on 4 April 1996 in the $304 \AA$ band and the averaged data obtained in the EIT 171,195 and $284 \AA$ bands.

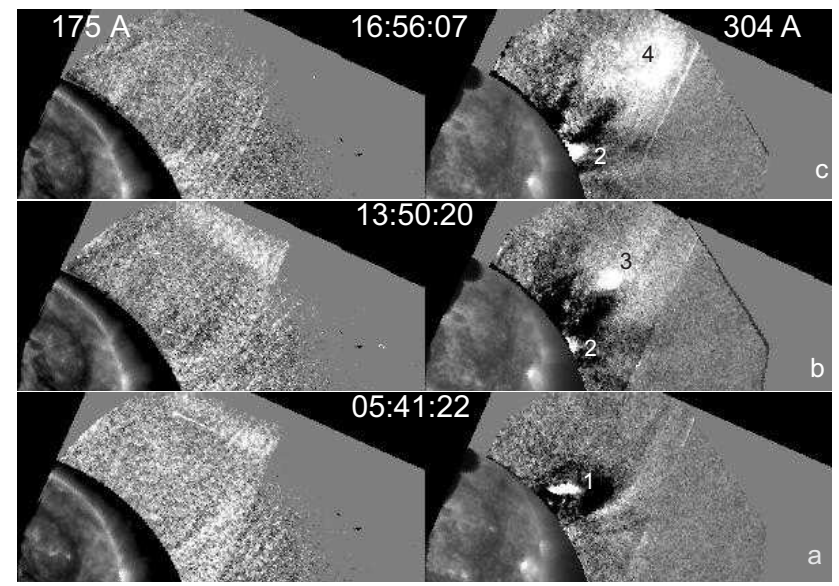

Fig. 10. Selected SPIRIT images of the failed prominence eruption and CME observed on 2 February 2003 at 05:41:22, 13:50:20 and 16:56:07 UT in the $175 \AA$ (left) and $304 \AA$ (right) bands: 1 - the prominence before eruption; 2 - a brightening after the failed eruption; 3, 4- different stages of the CME development. The coronal parts are shown as the base difference images with the reference image taken at 10:24:14 UT.

reference image taken at 10:24:14 UT. Earlier, from 1 February 16:00 UT to 2 February 10:00 UT, a slowly rising prominence appeared in $304 \AA$ as a bright feature (marked by the number 1 in Fig. 10a). Between 06:00 and 10:00 the prominence returned back and produced a brightening at the lower boundary of the FOV (the feature 2 in Fig. $10 \mathrm{~b}$ and c). After 13:29 UT a bright spot appeared at the distance $R \sim 1.5$ (the feature 3 ) which could indicate the beginning of the eruption 
process. At 16:56:07 this feature was transformed into a big, expanding loop (the feature 4 in Fig. 10c). After 18:30 the loop left the field of view at $R \sim 2.3$. The whole eruption process was seen only in the $304 \AA$ band and may be definitely related to the He II line, because in $175 \AA$, we can only see the traces of the rising loop. So we can conclude that the temperature of the erupting matter was sufficiently less than $1 \mathrm{MK}$. The LASCO CME catalogue described this event as a weak CME moving with the projected radial speed $V=165 \mathrm{Km} / \mathrm{s}$ (in the linear fit). It should be noted that in $304 \AA$, due to the Doppler dimming effect, we can detect only rather cold prominences and CMEs moving with the radial speed $V \leq 200 \mathrm{~km} / \mathrm{s}$ (Labrosse et al., 2006). Instead, in $175 \AA$ a detection of transients is limited only by the temperature conditions: the plasma temperature must be of the order of $1 \mathrm{MK}$.

\section{Conclusions}

The SPIRIT EUV telescope-coronagraph is proved to be an efficient instrument to study the solar corona and coronal transients from the solar limb to several solar radii. These data fill the gap between the images of the corona obtained by the ordinary EUV telescopes and WL coronagraphs. The instrument is efficient to detect CMEs, eruptive prominences and other slow moving transients. Depending to its temperature, they can be observed in $\mathrm{Fe}$ ion or $\mathrm{He}$ ion line bands.

Acknowledgements. The authors are very grateful to S. Koutchmy for his very important remarks and advises in preparing this work, and to J.-P. Delaboudinière for the useful discussions.

The work was partly supported by the grant of the Russian Foundation for Basic Research, project 05-02-17415, and the Program no. 16 of the Presidium of Russian Academy of Sciences.

The EIT, LASCO and UVCS data used here are produced by the SOHO Consortium.

SOHO is a project of international cooperation between ESA and NASA.

CHIANTI is a collaborative project involving the NRL (USA), RAL (UK), MSSL (UK), the Universities of Florence (Italy) and Cambridge (UK), and George Mason University (USA).

The authors and Topical Editor R. Forsyth thank L. Golub and another anonymous referee for their help in evaluating this paper.

\section{References}

Andretta, V., Del Zanna, G., and Jordan, S. D.: The EUV helium spectrum in the quiet Sun: a by-product of coronal emission, Astron. Astrophys., 400, 737-752, 2003.

Brueckner, G. E., Howard, R. A., Koomen, M. J., et al.: The Large Angle Spectroscopic Coronagraph (LASCO), Solar Phys., 162, 357-402, 1995.

Burkepile, J., Darnell, T., and Tomczyk, S.: Mauna Loa Solar Observatory and the SSSC Great Observatory, AGU Fall Meeting 2005, abstract \#SH51C-1220, 2005.
Cirtain, J., Martens, P. C. H., Acton, L. W., and Weber, M.: The EUV unresolved corona, Solar Phys., 235, 295-316, 2006.

DeForest, C. E.: On the size of structures in the solar corona, Astrophys. J., 661, 532-542, 2007.

Delaboudinière, J.-P., Artzner, G. E., Brunaud, J., et al.: EIT: Extreme-Ultraviolet Imaging telescope for the SOHO mission, Solar Phys., 162, 291-312, 1995.

Delaboudinière, J. P.: Resonance scattering of $30.4 \mathrm{~nm}$ chromospheric radiation by coronal singly ionized helium observed with EIT, Solar Phys., 188, 259-275, 1999.

Dere, K. P., Landi, E., Mason, H. E., Monsignori Fossi, B. C., and Young, P. R.: CHIANTI - an atomic database for emission lines, Astron. Astrophys. Suppl. Ser., 125, 149-173, 1997.

Judge, P. G. and Pietarila, A.: On the formation of extremeultraviolet helium lines in the Sun: analysis of SOHO data, Astrophys. J., 606, 1258-1275, 2004.

Kohl, J. L., Esser, R., Gardner L. D., et al.: The Ultraviolet Coronagraph Spectrometer for the Solar and Heliospheric Observatory, Solar Phys., 162, 313-356, 1995.

Koutchmy, S.: Coronal physics from eclipse observations, Adv. Space Res., 14, 29-39, 1994.

Koutchmy, S.: Coronal streamers, Encyclopedia of Astronomy and Astrophysics, edited by: Murdin, P., article 2271, Bristol, Institute of Physics Publishing, 2001.

Koutchmy, S. L. and Molodensky M. M.: Magnetic configurations of streamer structures in the solar atmosphere, Astronomy Lett., 31(6), 398-405, 2005.

Labrosse, N., Gouttebroze, P., and Vial, J.-C.: The Helium spectrum in erupting solar prominences, Highlights of Astronomy, edited by: van der Hucht, K. A., 14, 119-123, 2006.

Lamy, P., Quemerais, E., Liebaria, A., et al.: Electronic densities in coronal holes from LASCO C2 images, Proc. 5th SOHO Workshop "The Corona and Solar Wind near Minimum activity", Oslo, Norway, 17-20 June 1997, ESA SP-404, 491, 1997.

Li Jing, Kuhn, J., LaBonte, B., et al.: Global solar corona revealed by the time series observations, Astrophys. J., 538, 415-423, 2000.

Macpherson, K. P. and Jordan, C.: The anomalous intensities of helium lines in the quiet solar transition region, Mon. Not. R. Astron. Soc., 308, 510-526, 1999.

Parenti, S., Bromage, B. J. I., Poletto, G., et al.: Characteristics of solar coronal streamers. Element abundance, temperature and density from coordinated CDS and UVCS SOHO observations, Astron. Astrophys., 363, 800-814, 2000.

Quemerais, E. and Lamy, P.: Two-dimensional electron density in the solar corona from inversion of white light images - application to SOHO/LASCO-C2 observations, Astron. Astrophys., 393, 295-304, 2002.

Raymond, J. C., Kohl, J. L., Noci, G., et al.: Composition of coronal streamers from the SOHO Ultraviolet Coronagraph Spectrometer, Solar Phys., 175, 645-665, 1997.

Saez, F., Zhukov, A. N., Lamy, P., and Llebaria, A.: On the 3dimensional structure of the streamer belt of the solar corona, Astron. Astrophys., 442, 351-358, 2005.

Slemzin, V. A., Kuzin, S. V., Zhitnik, I. A., et al.: Observations of Solar EUV Radiation with the CORONAS-F/SPIRIT and SOHO/EIT Instruments, Solar Syst. Res., 39, 489-500, 2005.

Schwenn, R., Inhester, B., Plunkett, S. P., et al.: First view of the extended green-line emission corona at solar activity minimum 
using the LASCO C1 coronagraph on SOHO, Solar Phys., 175, 667-684, 1997.

Thompson, W. and Davila, J.: COR1 Current Status and Future Plans, presentation at the 5th SECCHI Consortium Meeting, Orsay, France, 5-8 March 2007.

Wang, Y.-M., Biersteker, J. B., Sheely, N. R., et al.: The solar eclipse of 2006 and origin of raylike features in the white-light corona, Astrophys. J., 660, 882-892, 2007.

Warren, H. P.: Measuring the physical properties of the solar corona: results from SUMER/SOHO and TRACE, Solar Phys., 190, 363-377, 1999.

Wilhelm, K., Marsch, E., Dwivedi, B. N., et al.: The solar corona above polar coronal holes as seen by SUMER on SOHO, Astrophys. J., 500, 1023-1038, 1998.
Wilhelm, K., Inhester, B., and Newmark, J. S.: The inner solar corona seen by SUMER, LASCO/C1 and EIT: electron densities and temperatures during the rise of the new solar cycle, Astron. Astrophys., 382, 328-341, 2002.

Wülser, J.-P., Lemen, J. R., Tarbell, T. D., et al.: EUVI: the STEREO-SECCHI extreme ultraviolet imager, Proc. SPIE, 5171, 111-122, 2004.

Zhitnik, I. A., Bougaenko, O. I., Delaboudinière, J.-P., et al.: SPIRIT x-ray telescope/spectroheliometer results, ESA SP-506 "Solar Variability: from Core to Outer Frontiers", 915-918, 2002.

Zhitnik, I., Kuzin, S., Afanas'ev, A., et al.: XUV observations of solar corona in the SPIRIT experiment on board the CORONASF satellite, Adv. Space Res., 32, 473-477, 2003.

Zirin, H.: The helium chromosphere, coronal holes, and stellar Xrays, Astrophys. J., 199, L63-L66, 1975. 